[Technical Paper]

\title{
Identifying EM Radiation from a Printed-Circuit Board Driven by Differential-Signaling
}

\author{
Yoshiki Kayano and Hiroshi Inoue \\ Akita University, 1-1 Tegata-Gakuen-machi, Akita 010-8502, Japan
}

(Received June 30, 2010; accepted September 30, 2010)

\begin{abstract}
Recently, differential-signaling (DS) techniques such as low-voltage differential signaling (LVDS) have come into wide use in digital electronics devices to suppress electromagnetic interference (EMI). In this paper, we attempted to newly identify the frequency responses of the EM radiation from a PCB driven by LVDS, using three EMI-antenna models. EM radiation is modeled and analyzed as EMI antennas, which depend on the configuration of the PCB. The first EMI antenna is a loop type due to the signal current flowing on the paired lines (EMI antenna I). The second EMI antenna is comprised of the ground plane and cable for a dipole type antenna due to a common-mode current flowing along the PCB with cable (EMI antenna II), and the third EMI antenna is comprised of the trace on the ground plane for the looptype antenna due to the signal current (EMI antenna III). It is demonstrated that the larger EMI antenna, which consists of the ground plane, is the dominant horizontal-component radiation factor at the lower frequencies. The proposed model can explain the characteristics of EM radiation from a PCB driven by differential signaling and also identify the primary radiation factor. The antenna model provides enough flexibility for different geometrical parameters and increases our ability to provide insight and design guidelines.
\end{abstract}

Keywords: Electromagnetic interference, Electromagnetic radiation, Common-mode, Differential-mode, Differential-signaling

\section{Introduction}

In recent years, the increasing clock frequency and packaging density of electronic devices have resulted in more potential noise interference and radiation issues. All electronic devices must satisfy the requirements of electromagnetic compatibility (EMC) for low electromagnetic interference (EMI).[1] Hence, effective methods for predicting and suppressing EMI as well as maintaining signal integrity over a broad band are required.

So far, some studies on the EM radiation from printed circuit boards (PCBs) have been published,[2-6] with the discussion on two radiation components, common-mode (CM) and differential-mode (DM). Although the DM current is the desired current for the operation, the $\mathrm{CM}$ current is an imbalance component and is not intended to be present, but exists in practical systems. Although the CM current is a very significant and classical problem, ideal models such as the transmission-line model cannot predict the $\mathrm{CM}$ current. Mechanisms by which the signal current is converted to CM-noise sources resulting in EMI have been demonstrated for frequencies lower than the first resonant frequency in Ref..[3] The authors have discussed the $\mathrm{CM}$ current on a feed cable due to a trace near a PCB edge and a prediction method for EM radiation by experimental and numerical methods. $[7,8]$ It is necessary to suppress the $\mathrm{CM}$ current to reduce the EM radiation.

Recently, differential-signaling (DS) techniques such as low-voltage differential signaling (LVDS) are widely used in digital electronic devices to suppress the EMI.[9-15] Improvements of signal integrity (SI) performance such as cross-talk noise and eye-diagram have been reported. In addition, the suppression effect of the differentialtransmission mode on EM radiation has been demonstrated.[14] Many papers have demonstrated that imbalances in differential signals deteriorate EMI and SI performance. But investigations of the frequency response of EMI have not been thoroughgoing enough. It is helpful to be able to anticipate at the design stage the EMI frequency 
response from the $\mathrm{PCB}$ configuration.

In this paper, we attempt to newly identify the frequency responses of the $\mathrm{EM}$ radiation from a $\mathrm{PCB}$ driven by LVDS, using three EMI-antenna models. To provide basic considerations for suppressing the EM radiation, the $\mathrm{CM}$ current and far-electric field are investigated experimentally and theoretically with finite-difference time-domain (FDTD) modeling. Firstly, the PCB geometry used in the experimentation and the three EMI-antenna models are explained in Section 2. Secondly, the experimental setups, and the conditions of the FDTD modeling are described in Section 3. In Section 4, the effect of the PCB dimension on EMI is discussed by changing the PCB geometry. Specifically, the focus is on the composition of the EMI antenna. Finally, the frequency response of EMI is identified.

\section{PCB Geometry and EMI Antenna Model}

\subsection{Model}

The geometry of the PCB under study is illustrated in Fig. 1. The PCB has two layers, with the upper layer for

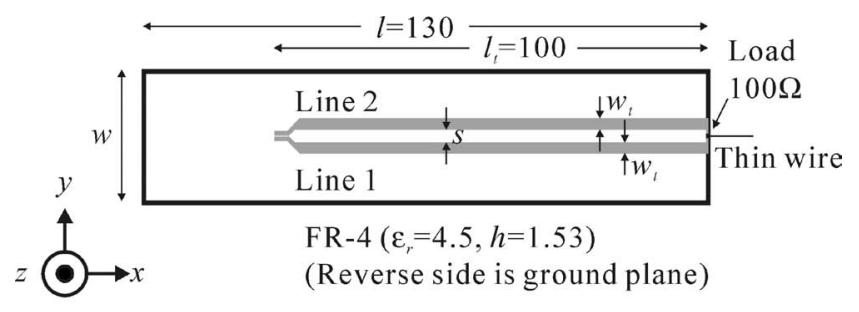

[in $\mathrm{mm}$ ]

(a) Top view

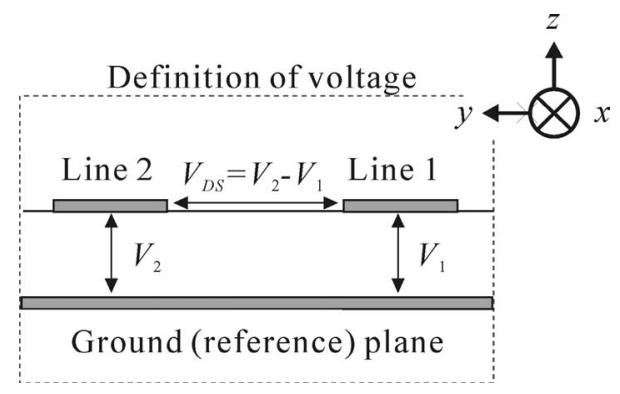

(b) Definition of the voltage

Fig. 1 Geometry of the PCB under study.

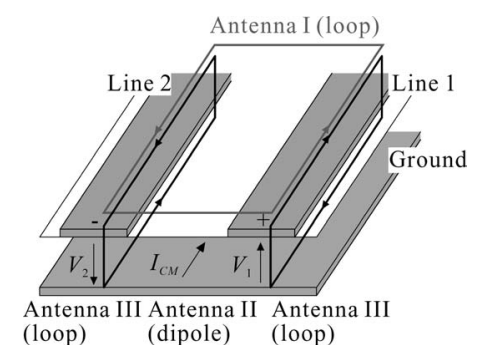

Fig. 2 Three EMI-antenna models explaining EM radiation from a differential pair structure. the signal trace and the lower layer for the reference (ground) plane. Two radiation components, CM and DM, are the main sources of the far-electric field radiated from the model PCB. In order to explain the characteristics of EM radiation from a $\mathrm{PCB}$ driven by differential signaling, three EMI-antenna models are proposed. The three models are shown in Fig. 2. The first EMI antenna is a loop type due to the signal current flowing on the paired lines (EMI antenna I). The second EMI antenna is comprised of the ground plane and cable for a dipole type antenna due to a $\mathrm{CM}$ current flowing along the PCB with cable (EMI antenna II), and the third EMI antenna is comprised of the trace on the ground plane for the loop-type antenna due to a signal current (EMI antenna III). There are two CM components flowing along the PCB. One is due to the asymmetrical voltage waveforms on lines 1 and 2 . The other is due to the non-zero impedance of the ground plane, which has a finite width, and has magnetic flux lines that close below the ground plane.[3] EMI antennas I and II radiate the horizontal component. On the other hand, EMI antenna III radiates the vertical component.

\subsection{PCB configuration}

The size of the microstrip line structure used for the test model is $l=130 \mathrm{~mm}, w=30,60$ and $90 \mathrm{~mm}$, and $h=1.53$ $\mathrm{mm}$ of the dielectric substrate with a permittivity of $\varepsilon_{r}=$ 4.5. Two traces with $100 \mathrm{~mm}$ length, width $w_{t}$ and distance $s$ are located on the dielectric substrate. To discuss and demonstrate the effect of the configuration of the PCB on $\mathrm{EM}$ radiation, some different configurations, shown in Table 1, were prepared. For impedance matching, the $w_{t}$ and $s$ are designed so that the differential-mode impedance of the paired lines is $Z_{D M}=100 \Omega$, and the traces are terminated with $100 \Omega$. The differential paired lines are driven by an LVDS driver (NS DS90LV047A), and a crystal oscillator with $3.3 \mathrm{~V}$ amplitude and $33.333 \mathrm{MHz}$ oscillation frequency. The crystal oscillator, LVDS driver, and battery are installed on the reverse side, and are covered with a

Table 1 PCB under study.

\begin{tabular}{|c|c|c|c|c|c|}
\hline$s[\mathrm{~mm}]$ & $w_{t}[\mathrm{~mm}]$ & $Z_{D M}[\Omega]$ & $\varepsilon_{\text {eff } D M}$ & $Z_{C M}[\Omega]$ & $\varepsilon_{\text {eff } C M}$ \\
\hline 0.3 & 1.0 & \multirow{5}{*}{100} & 2.79 & 55.1 & 3.32 \\
\hline 1.0 & 1.9 & & 2.90 & 36.3 & 3.51 \\
\hline 3.0 & 2.5 & & 3.09 & 26.2 & 3.55 \\
\hline 5.0 & 2.7 & & 3.18 & 26.2 & 3.51 \\
\hline 10.0 & 2.8 & & 3.27 & 25.2 & 3.44 \\
\hline
\end{tabular}

$w=30,60$ and $90 \mathrm{~mm}$ 
shield box (thin-sheet copper).

To demonstrate the advantages of differential signaling, the EM radiation from a single-end type $\mathrm{PCB}$ is compared with the EM radiation from the PCB driven by the LVDS. The size of the single-end type PCB is identical to that of the PCB driven by the LVDS. The trace, with a $2.8 \mathrm{~mm}$ width, $50 \Omega$ characteristic impedance, and $100 \mathrm{~mm}$ length, is centered on the dielectric with a permittivity of $\varepsilon_{r}=4.5$, and terminated with a $50 \Omega$ resistor. The signal trace is driven by a crystal oscillator with a $3.3 \mathrm{~V}$ amplitude and 33.333 $\mathrm{MHz}$ oscillation frequency. The far-electric field components radiated from a single-ended PCB are identified by EMI antennas II and III.

Figure 3(a) shows the waveforms of voltage $V_{1}$ and $V_{2}$ against the ground, and the differential voltage $V_{D S}$ measured at the input terminal of the signal lines for $s=3.0$ $\mathrm{mm}$ and $w=30 \mathrm{~mm}$. Figure 3 (b) shows the frequency response of the differential voltage $V_{D S}$. Voltages traveling along line 1 and line 2 are asymmetrical waveforms due to the differences of phase-shift and amplitude. Hence, an
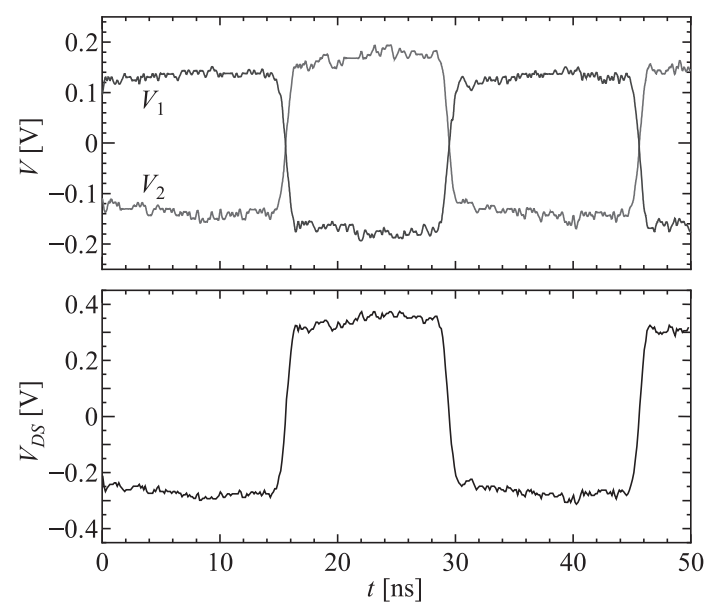

(a) Waveforms

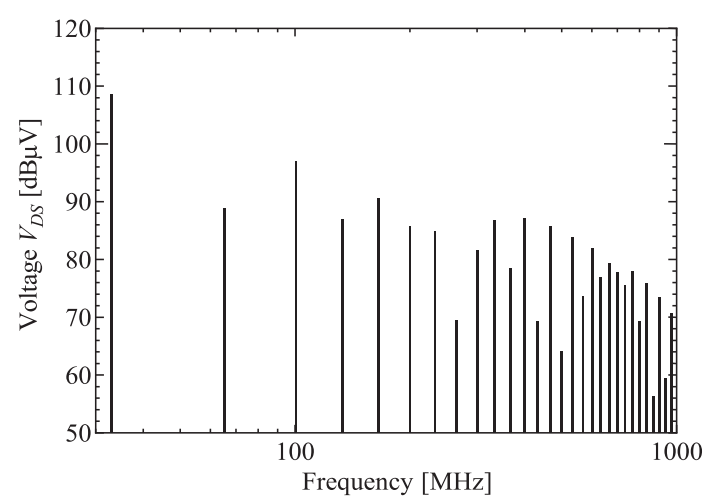

(b) Frequency response

Fig. 3 The line voltage $V$ and differential voltage $V_{D M}$ measured at the input terminal of the signal line in the case of $s=$ $3.0 \mathrm{~mm}$ and $w=30 \mathrm{~mm}$. imbalanced component arises.

\section{Experimental and Numerical Modeling Methods}

\subsection{Experimental setup}

The CM current at the connecting point of the thin wire attached to the ground plane was measured by a current probe (Fischer F-2000), which encircles the wire, and a spectrum analyzer (Agilent E4402B). The CM current, $I_{C M}$, was obtained from the input voltage $V_{i n}$ of the spectrum analyzer and the transfer impedance, $Z_{T}$, of the current probe.

The far-electric field radiated from the PCB was measured in an anechoic chamber with a Bi-Log antenna (30$1000 \mathrm{MHz}$ ), as shown in Fig. 4. The coordinate system is also notated, where $\phi$ is the counterclockwise angle in the $x-y$ plane measured from the positive $x$-axis, and $\theta$ is the angle from the positive $z$-axis. The PCB was placed on a wooden Table. The distance between the PCB and the antenna was $3 \mathrm{~m}$. The RBW and VBW of the spectrum analyzer are $5 \mathrm{kHz}$. The horizontal component $E_{\phi}$, at $(\phi, \theta)=$ $\left(90^{\circ}, 90^{\circ}\right): E_{x}$ and the vertical component, $E_{\theta}$, at $(\phi, \theta)=$ $\left(90^{\circ}, 90^{\circ}\right): E_{z}$ are discussed.

\subsection{Method of FDTD modeling}

The FDTD method[16] is used for the near field simulation, and calculation of the far-electric fields. The FDTD model provides results for "ideal balanced differentialsignaling $\left(V_{2}=-V_{1}\right)$ ". The FDTD modeling details were determined by varying the number of cells for trace width, substrate thickness, and the space between the PCB and the absorbing boundary condition. A cell size of $\Delta x=2.0$, $\Delta y=0.1$, and $\Delta z=0.51 \mathrm{~mm}$ was determined from our former studies. The time step was $\Delta t=0.326 \mathrm{ps}$ from the Courant stability condition.[16] Perfectly matched layers (PMLs),[17] twelve cells deep, were used as the absorbing boundary condition. The trace and ground plane were modeled as a perfect electric conductor (PEC). The PCB substrate was modeled as a dielectric three cells deep with a relative permittivity $\varepsilon_{r}=4.5$. The source was modeled as

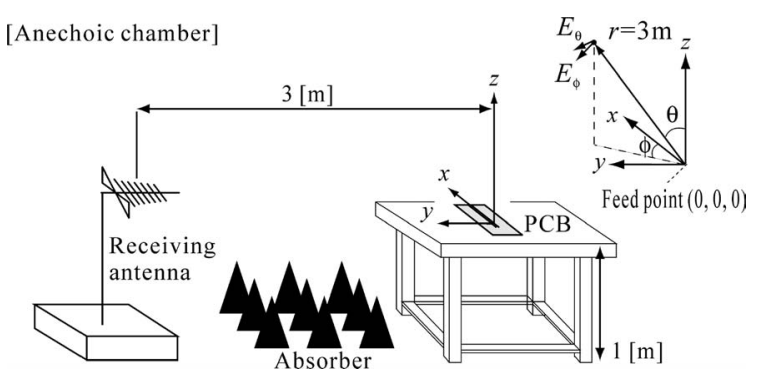

Fig. 4 Experimental setup for far-electric field measurement. 
a voltage source with an internal resistance of $50 \Omega$. A sinusoidally modulated Gaussian pulse voltage with a frequency range from $30 \mathrm{MHz}$ to $3 \mathrm{GHz}$ was applied as the signal waveform.

The equivalent electric and magnetic current distributions were determined from the calculated values of the electric and magnetic fields on the closed surface surrounding the FDTD model. The far-electric field was obtained using the near-to-far-field transformation from the surface electric and magnetic current distributions.[18]

\section{Results and Discussion}

\subsection{EMI antenna I}

To identify EMI antenna I, the effect of the distance, $s$, between the differential paired lines on the EMI is discussed. To clarify the frequency response, the measured results with harmonics of $33.333 \mathrm{MHz}$ are normalized to the case where the frequency response of the input voltage to the trace is $0 \mathrm{dBm}$ constant. The frequency responses of the normalized CM current and the horizontal component of the far-electric field with different distances are shown in Fig. 5. The symbols and lines are the measured and calculated results, respectively. The measured results which are smaller than the noise floor level are omitted in Fig. 5. As the distance between the differential paired lines decreases, the CM current and far-electric field decrease. The results indicate that DS is effective in suppressing EM radiation. As the frequency becomes higher, EM radiation increases.

Good agreement between the calculated and the measured results up to $500 \mathrm{MHz}$ shows the validity of the numerical results. On the other hand, the measured results for the LVDS (DS: $s=0.3,3.0$ and $10.0 \mathrm{~mm}$ ) cases are larger than the calculated results above $500 \mathrm{MHz}$. Although the results shown in Fig. 5(b) do not allow distinguishing between the radiation components from antennas I and II, this fact indicates that the EM radiation due to the unintentional imbalanced component is the dominant factor of high frequency EMI.

\subsection{EMI antenna II}

To identify EMI antenna II, the effect of the width, $w$, of the ground plane on EMI is discussed. The frequency responses of the normalized CM current and the horizontal component of the far-electric field with different ground plane widths are shown in Fig. 6. As the width of the ground plane becomes narrower, the $\mathrm{CM}$ current and

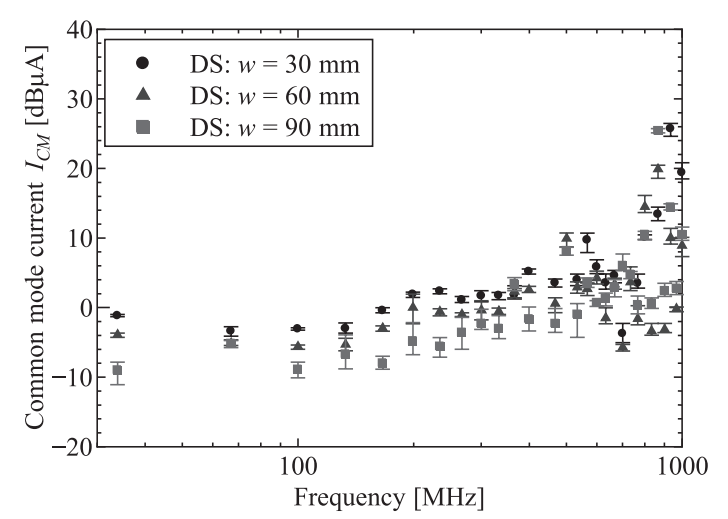

(a) $\mathrm{CM}$ current

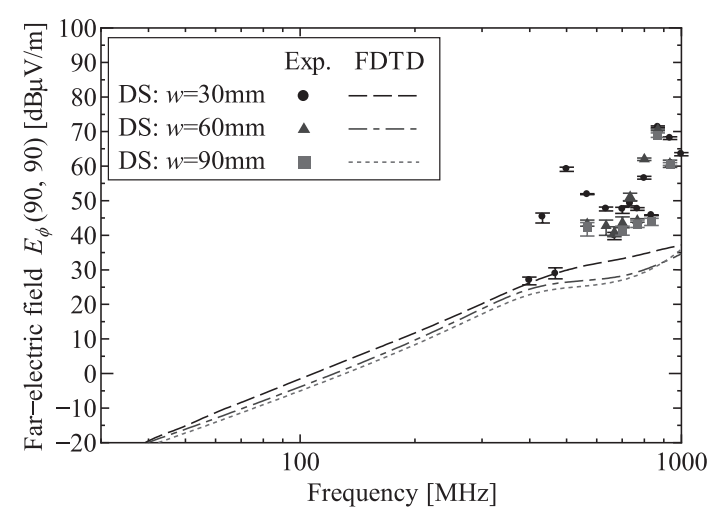

(b) Horizontal component of the far-electric field

Fig. 6 Frequency response of the EMI with different ground plane width $w(s=0.3 \mathrm{~mm})$.
Fig. 5 Frequency response of the EMI with different distance $s(w=30 \mathrm{~mm})$. 


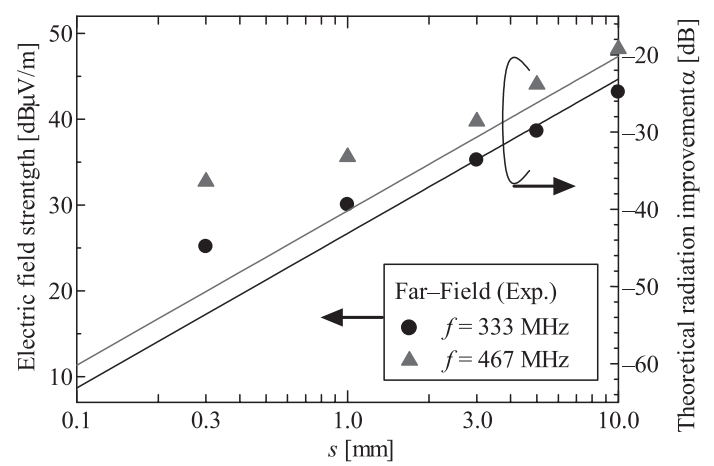

Fig. 7 Effect of distance $s$ between differential paired lines on $E_{x}(w=30 \mathrm{~mm})$.

horizontal components increase. The measured results are much larger than the calculated results. This result indicates that the horizontal component in the close condition of $s=0.3 \mathrm{~mm}$ is dominated by the imbalanced current due to the asymmetrical waveform.

To understand the details of the imbalanced component, the effect of the distance, $s$, between differential paired lines on $E_{x}$ are discussed. Figure 7 shows the relationship between $s$ and $E_{x}$ and a theoretical improvement $\alpha$ with differential signaling, where $\alpha$ is defined as the ratio of the far-electric field radiated from differential paired lines to that from a single-end microstrip line:

$$
\alpha \equiv \frac{E_{D S}}{E_{\text {single-end }}}=1-\frac{r}{r+s_{c}} e^{-j \frac{2 \pi s_{c}}{c_{0}}},
$$

where $s_{c}$ is the distance between the centers of the two traces, $s_{c}=s+w_{t}, c_{0}$ is the speed of light, and $r$ is the observation point of the far-electric field.[13] As the distance between differential paired lines decreases, $\alpha$ decreases. When they are widely separated $(s>3.0 \mathrm{~mm}), E_{x}$ corresponds to $\alpha$. Thus, the uncanceled differential component is the dominant factor of EM radiation. However, when the lines are narrowly separated $(s<3.0 \mathrm{~mm}), E_{x}$ does not follow the theoretical improvement curve. The CM component of the total EM radiation can no longer be disregarded compared with the differential component. The voltage waveforms on the differential paired lines must be balanced to better than $0.1 \%$ to achieve $\alpha$ of $-60 \mathrm{~dB}$.[13] These results indicate that EM radiation for $s<3.0 \mathrm{~mm}$ is dominated by the $\mathrm{CM}$ component due to the asymmetrical waveform.

\subsection{EMI antenna III}

To identify EMI antenna III, the effect of the distance, $s$, between differential paired lines on the vertical component, $E_{z}$, is discussed. The frequency responses of nor-

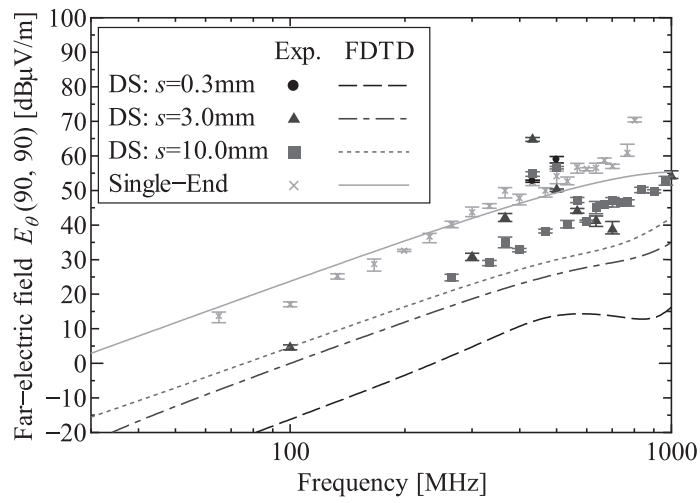

Fig. 8 Frequency response of vertical component with different distance $s(w=30 \mathrm{~mm})$.

malized far-electric fields for various distances are shown in Fig. 8. As the distance between the paired lines decreases, the vertical components, $E_{z}$, decrease. In the "DS" case, the measured results are larger than the calculated results. These consequences indicate that the difference between the measured and the calculated results on the vertical component is caused by imbalanced current due to the asymmetrical waveform. Consequently, correct estimation of the imbalance due to the asymmetrical waveform is key to the prediction of the total EMI behavior of the PCB driven by LVDS.

\subsection{Discussion on imbalanced component}

To understand the details of the imbalanced component, the distribution and frequency response of a magnetic near field are discussed. Figure 9 shows the distribution of the magnetic field, $H_{y}$, along the $y$ direction about $1.0 \mathrm{~mm}$ above the differential paired lines. A magnetic near-field is mainly distributed oppositely surrounding each trace because of differential signaling. The calculated results are ideally balanced. Although the measured and calculated results at $f=33.333 \mathrm{MHz}$ are in good agreement, the measured result at $f=1000 \mathrm{MHz}$ shows an asymmetrical distribution and hence an imbalanced component exists. Such an imbalanced current causes significant EM radiation.

Figure 10(a) shows the frequency response of the magnetic near field, $H_{y}$, on the differential paired lines. Figure 10 (b) shows the frequency response of the difference, $\Delta H_{y}$, between the magnetic near fields on lines 1 and 2. $\Delta H_{y}$ is relatively large above $500 \mathrm{MHz}$, and hence the imbalanced component of the total EM radiation can no longer be disregard. These results indicate that the difference between the measured and the calculated results on the far-electric field above $500 \mathrm{MHz}$ is caused by imbalanced current. 


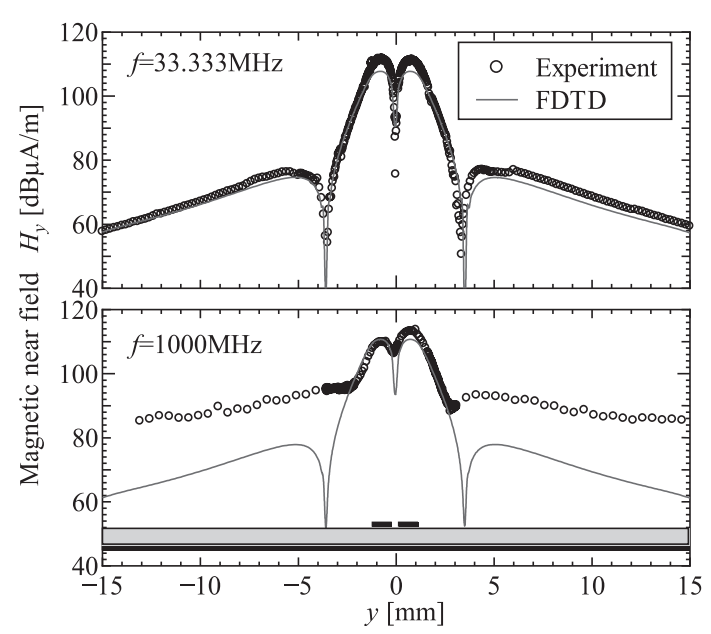

(a) $s=0.3 \mathrm{~mm}$

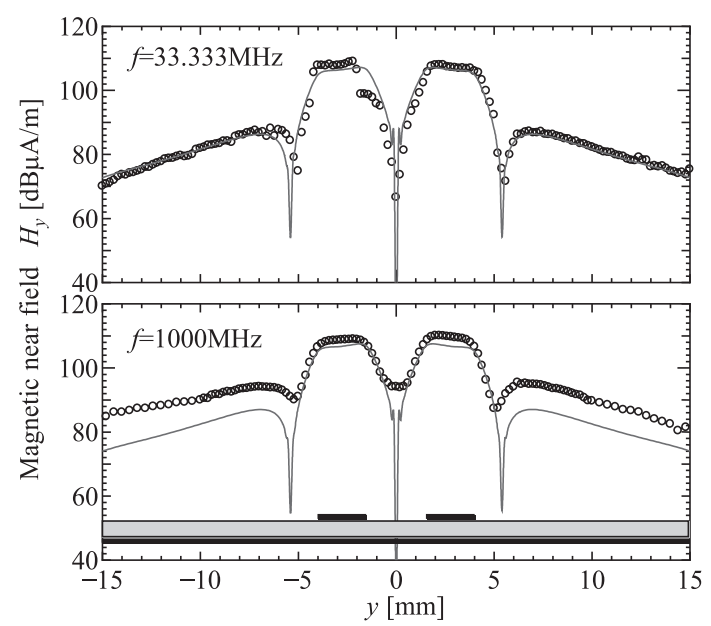

(b) $s=3.0 \mathrm{~mm}$

Fig. 9 Magnetic near field $H_{y}$ distribution along the $y$ direction at $1.0 \mathrm{~mm}$ above the differential paired lines. $(w=30 \mathrm{~mm})$.

\subsection{Discussion on frequency response of EM radia- tion}

Although the CM current is smaller than the DM current, a slight $\mathrm{CM}$ current can dominate total $\mathrm{EM}$ radiation because the radiation efficiency of EMI antenna II is much higher than that of EMI antenna I. Therefore, the horizontal component is dominated by EMI antenna II. The larger EMI antenna of the ground plane is the dominant radiation factor in the lower frequencies. Since the efficiency of $\mathrm{CM}$ radiation is proportional to the frequency, the far electric field follows $40 \mathrm{~dB} /$ decade at lower frequencies. The first resonant frequency is $880 \mathrm{MHz}$, which is related to the half-wavelength resonance of the ground dipole antenna. Consequently, it is possible that the outline of the frequency response of the undesired EM radiation from the PCB driven by LVDS was identified. Predicting the dominant component in certain frequency bands is very helpful in curbing EMI problems in high-speed electronic designs.

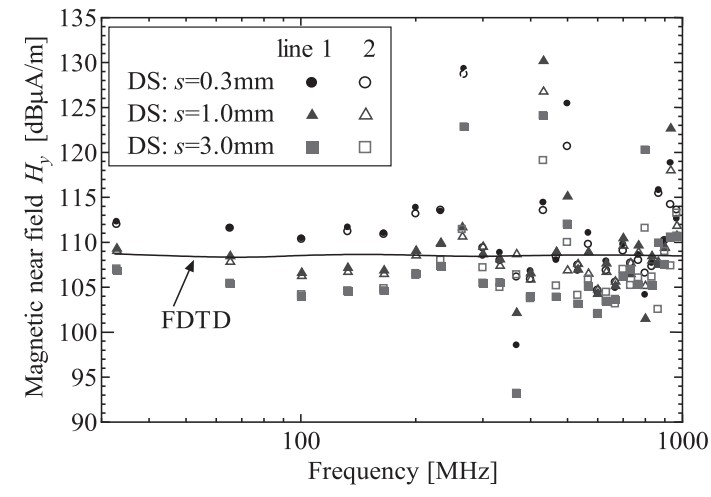

(a) $H_{y}$

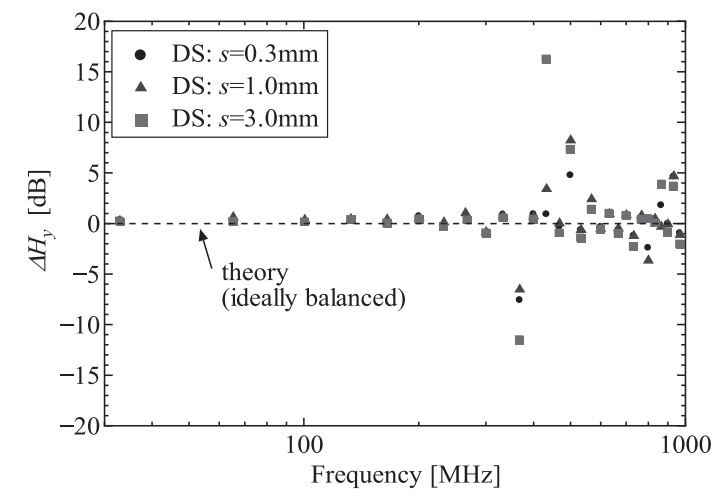

(b) $\Delta H_{y}$

Fig. 10 Frequency response of magnetic near field $H_{y}$.

\section{Conclusion}

The EM radiation from a PCB driven by LVDS was modeled and analyzed as EMI antennas, which depend on the configuration of the PCB. Three EMI-antenna models were proposed and demonstrated to identify the EMI frequency responses. It was demonstrated that the larger EMI antenna, which consists of the ground plane, is the dominant radiation factor in the lower frequencies. The proposed model can explain the characteristics of EM radiation from a PCB driven by differential signaling and also identify the primary radiation factor. The antenna model provides enough flexibility for different geometrical parameters and increases our ability to provide insight and design guidelines.

Easier interpretations for the generation of imbalanced currents are required to predict and suppress the EMI. Further work will be carried out on this concept.

\section{Acknowledgements}

The authors express their thanks to the Akita Research Institute of Advanced Technology, Mr. Michiaki Nakano, Mr. Yusei Ono and Mr. Ryosuke Hashiya of our research laboratory, for their support of measurements, and the Cyberscience Center, Tohoku University, and the General 
Information Processing Center, Akita University, for their support with computer resources. This research was partially supported by the Ministry of Education, Science, Sports and Culture, Grant-in-Aid for Young Scientists (B), 19760190, Akita University Researcher Overseas Transfer Program in 2008, and TELECOM ENGINEERING CENTER.

\section{References}

[1] C. R. Paul, Introduction to Electromagnetic Compatibility, John Wiley \& Sons, New York, 1991.

[2] F. B. J. Leferink, "Signal to Noise Transformation, the Key to EMC," in Proc. IEEE Int. Symp. Electromagn. Compat., Chicago, IL, pp. 462-467, 1994.

[3] D. M. Hockanson, J. L. Drewniak, T. H. Hubing, T. P. VanDoren, F. Sha, and M. J. Wilhelm, "Investigation of Fundamental EMI Source Mechanisms Driving Common Mode Radiation from Printed Circuit Boards with Attached Cables," IEEE Trans. Electromagn. Compat., Vol. 38, no. 4, pp. 557-566, Nov. 1996.

[4] T. Watanabe, O. Wada, T. Miyashita, and R. Koga, "Common-Mode-Current Generation Caused by Difference of Unbalance of Transmission Lines on a Printed Circuit Board with Narrow Ground Pattern," IEICE Trans. Commun., Vol. E83-B, No. 3, pp. 593599, Mar. 2000.

[5] I. F. Chen and C. W. Hsue, "Evaluation of CommonMode Radiation from Printed Circuit Boards by Modelling Imperfect Ground Effect," IEICE Trans. Commun., Vol. E85-B, No. 12, pp. 2924-2933, Dec. 2002.

[6] H. Sasaki, T. Harada, and T. Kuriyama, "Dependence of Signal Trace Layout on Emissions Radiated from Printed Circuit Board," IEICE Trans. Commun. (Japanese Ed.), Vol. J90-B, No. 11, pp. 1124-1134, Nov. 2007.

[7] Y. Kayano, M. Tanaka, J. L. Drewniak, and H. Inoue, "Common-Mode Current Due to a Trace Near a PCB Edge and Its Suppression by a Guard Band,” IEEE Trans. Electromagn. Copat., Vol. 46, No. 1, pp. 46-53, Feb. 2004.

[8] Y. Kayano and H. Inoue, "Prediction of EM Radia- tion from a PCB Driven by a Connected Feed Cable,” IEICE Trans. Commun., Vol. E92-B, No. 6, pp. 1920-1928, Jun. 2009.

[9] S. Hall, G. W. Hall, and J. A. McCall, High-Speed Digital System Design: A Handbook of Interconnect Theory and Design Practices, John Wiley \& Sons, INC., New York, 2000.

[10] J. Kudo and T. Sudo, "Evaluation of Propagation Characteristics in High-Speed Differential Signal Pair,” in Proc. ICEP, Tokyo, Japan, pp. 57-62, 2002.

[11] M. Leone and V. Navratil, "On the External Inductive Coupling of Differential Signaling on Printed Circuit Boards," IEEE Trans. Electromagn. Compat., Vol. 46, No. 1, pp. 54-61, Feb. 2004.

[12] A. Tanaka, "Practical Side of PCB Pattern Design Corresponding to High-Speed Differential Transmission,” Journal of JIEP, Vol. 8, No. 4, pp. 271-276, 2005.

[13] H. Johnson and M. Graham, High-Speed Signal Propagation: Advanced Black Magic, Prenticel Hall, NJ, 2003.

[14] R. Matsubara, K. Miyauchi, Y. Ishida, M. Tokuda, and N. Kuwabara, "Investigation on Suppression Effect of Radiated Electromagnetic Field from Printed Circuit Board with Differential Transmission Mode Using Method of Moments," IEICE Trans. Commun. (Japanese Ed.), Vol. J87-B, No. 1, pp. 1926-1935, Nov. 2004.

[15] A. Takagi, "EMI Reducing Techniques for Low Voltage Differential Signaling on a Flexible Printed Circuit Board," IEICE Trans. Electron. (Japanese Ed.), Vol. J87-C, No. 11, pp. 918-927, Nov. 2004.

[16] A. Taflove, Computational Electrodynamics: The Finite-Difference Time-Domain Method, Artech House, Norwood, MA, 1995.

[17] J. P. Berenger, "Three-Dimensional Perfectly Matched Layer for the Absorption of Electromagnetic Waves,” J. Comput. Phys., Vol. 127, pp. 363379, 1996.

[18] R. J. Luebbers, K. S. Kunz, M. Schneider, and F. Hunsberger, "A Finite-Difference Time Domain Near to Far-Zone Transformation," IEEE Trans. Antennas Propag., Vol. 39, No. 4, pp. 429-433, Apr. 1991. 